La revue La revue pour l'histoire du CNRS

POUR L'HISTOIRE DU CNRS

$22 \mid 2008$

La géopolitique mode d'emploi

\title{
Le Golan au cœur de la géopolitique d'Israël
}

Frédéric Encel

\section{OpenEdition}

Journals

Édition électronique

URL : https://journals.openedition.org/histoire-cnrs/8242

DOI : 10.4000/histoire-cnrs.8242

ISSN : 1955-2408

Éditeur

CNRS Éditions

Édition imprimée

Date de publication : 3 octobre 2008

ISBN : 978-2-271-06676-3

ISSN : 1298-9800

\section{Référence électronique}

Frédéric Encel, «Le Golan au cœur de la géopolitique d'Israël », La revue pour l'histoire du CNRS [En

ligne], 22 | 2008, mis en ligne le 03 octobre 2010, consulté le 20 mai 2021. URL : http://

journals.openedition.org/histoire-cnrs/8242 ; DOI : https://doi.org/10.4000/histoire-cnrs.8242

Ce document a été généré automatiquement le 20 mai 2021.

Comité pour l'histoire du CNRS 


\title{
Le Golan au cœur de la géopolitique d'Israël
}

\author{
Frédéric Encel
}

Une question de représentations

1 L'Israélien moyen - appelons-le l'homme de la rue à Tel Aviv - plutôt laïc, votant au centre gauche travailliste ou au centre droit Likoud selon les circonstances, mais en tout cas attaché au concept de "pureté des armes»(toar haneshek), distingue très nettement son compatriote installé sur le plateau du Golan de celui implanté en JudéeSamarie (Cisjordanie) centrale ou (jusqu'au retrait de 2005) à Gaza. Un différentiel de représentation qui commence par une distinction sémantique: les mityashvim, littéralement les installés, désignent les Israéliens du Golan et - dans une moindre mesure - ceux de la vallée du Jourdain et du Goush Etzion. Ces deux dernières zones se situent certes en Cisjordanie, mais hors des fortes densités de population arabes. Ainsi, la variable principale de désignation positive ne correspond pas au degré de sacralité de la terre, mais bien à l'absence de problème moral lié à l'absence de représentation visuelle d'une occupation. Tandis que les mitnakhalim ${ }^{1}$, kippa vissée sur le crâne et revolver au ceinturon, patrouillent aux abords de camps de réfugiés palestiniens en ébullition, les mityashvim en short et bob cultivent des fruits et des fleurs. Pendant que les premiers prient et profèrent des harangues messianistes, les seconds chantent des chants folkloriques et abreuvent le pays des bienfaits de leurs produits naturels.

2 C'est schématiquement ainsi que depuis les années 1970, le mabat, populaire journal télévisé de 20 heures - ainsi que de multiples émissions - présentent deux réalités dissemblables ${ }^{2}$. Et lorsque l'israélien se fait touriste, la télévision comme viatique est même dépassée par l'expérience du terrain : terrain inquiétant voire dangereux sur les routes de la dorsale cisjordanienne qu'on emprunte pour aller visiter des parents, terrain absolument sécurisé sur un Golan seulement peuplé de compatriotes juifs ; en outre, même demeurés syriens, les Druzes des quatre bourgs accrochés aux pentes du Hermon ne suscitent aucune crainte ${ }^{3}$. Quant au juif israélien plus conservateur voire religieux, il trouve dans le Golan un territoire biblique (le Bashan fertile) serti de vestiges de synagogues antiques, de pièces de monnaie battues à l'effigie de rois juifs, 
de traces de luttes héroïques contre l'envahisseur romain (Gamla), etc. ${ }^{4}$ Il n'est pas jusqu'au socle commun du recueillement sur les mémoriaux de la guerre du Kippour qui ne joue en faveur de l'engouement national ; sauf les ultra-orthodoxes, chaque juif israélien connaît un proche qui y a combattu s'il n'a combattu lui-même, et tout homme y a au moins crapahuté pendant son long et éprouvant service national et/ou durant ses périodes de réserve.

3 Enfin, la représentation de l'ennemi achève le tableau : par rapport aux autres voisins arabes, les Syriens pâtissent en Israël d'une réputation de cruauté, d'intransigeance et d'animosité antisémite sans égale ${ }^{5}$. Et les Israéliens d'accorder ainsi avec d'autant plus de sincérité un rôle stratégique à leurs compatriotes implantés face à ce péril permanent. Pour une majorité d'entre eux, ceux ne se reconnaissant pas dans le mouvement sioniste-religieux d'essence nationaliste, les mitnakhalim ne défendent Israël - à l'extrême limite - que contre les terroristes, en période d'Intifada. Bien souvent, lorsque la région connaît une baisse de tension, cette perception en mi-teinte laisse place à celle des mitnakhalim exaspérant les Palestiniens. Les mityashvim du Golan bénéficient en revanche d'une image de sentinelles courageuses face au plus implacable des ennemis étatiques d'Israël, la Syrie. C'est manifestement là que les réalités objectives s'écartent le plus des représentations : les guerres modernes rendent en effet tout à fait dérisoires l'intérêt stratégique de quelques points de peuplement civils; en octobre 1973 déjà, ceux-ci avaient dû être évacués en hâte devant la déferlante des blindés syriens ${ }^{6}$.

4 De fait, si la bande de Gaza et quatre implantations de Cisjordanie ont été évacuées, en 2005, avec l'approbation d'une opinion publique soutenant par ailleurs, sondage après sondage, l'évacuation d'autres implantations de mitnakhalim, le Golan recueille un plébiscite permanent. Depuis la conquête du plateau par Tsahal le 10 juin 1967, jamais un scrutin, une enquête d'opinion, un vote parlementaire ou une manifestation de rue n'a traduit le souhait de sa rétrocession par une majorité d'Israéliens, même relative ${ }^{7}$. En 1994-95 et 1999-2000, quand les gouvernements Rabin puis Barak tentèrent de négocier le Golan avec le régime damascène, des manifestations monstre ainsi qu'une scission au parti travailliste (la Troisième voie, du héros de la guerre du Kippour Avigdor Kahalani) réaffirmèrent de façon spectaculaire cet engouement ${ }^{8}$. La loi d'annexion du 14 décembre 1981 fut du reste renforcée le 26 janvier 1999 par une loi sans précédent; tout gouvernement décidant de compromis sur le plateau devra dorénavant réunir une majorité qualifiée de 70 députés à la Knesset ou de $65 \%$ des suffrages lors d'un référendum.

Une question de géographie, celle qui « sert d'abord à faire la guerre »

Contrefort méridional de la chaîne montagneuse de l'anti-Liban, le plateau du Golan est une formation volcanique couverte de roches basaltiques, profondément accidentée par des oueds engorgés entre des versants escarpés, s'étendant sur moins de $1160 \mathrm{~km}$ pentes abruptes du mont Hermon comprises - soit à peine l'équivalant du département français du Val d'Oise. Il se présente très approximativement sous une forme ovale, avec pour largeur entre 12 et $25 \mathrm{~km}$, et pour longueur maximale $67 \mathrm{~km}$. Si la superficie de ce territoire est donc extrêmement modeste, même à l'échelle des États de petite taille qui le jouxtent (Israël, Syrie, dans une moindre mesure Liban et Jordanie), sa topographie particulière lui confère l'essentiel de sa valeur stratégique. Sur un axe approximatif sud-ouest/nord-est, le Golan s'élève assez régulièrement en une courbe convexe jusqu'au massif du Hermon. À l'ouest, côté israélien, de profondes failles 
d'escarpement se traduisent par un dénivelé spectaculaire en surplomb de l'étroite vallée du Hulé, laquelle abrite le cours supérieur du Jourdain, et du Lac de Tibériade. La ligne de crête qui domine ces espaces s'élève jusqu'à la cote 700.

6 À l'est, côté syrien, le plateau accuse une pente moins prononcée que sur son versant occidental, et meurt dans la plaine du Hauran qui le sépare de Damas sur moins de 50 $\mathrm{km}$. Par ailleurs une série de cônes volcaniques, sortes de hautes levées de terre noire dont certaines dépassent 800 mètres de hauteur, s'élève en un axe nord-sud sur la ligne approximative de partage des eaux. Ces protubérances naturelles ne constituent pas des obstacles susceptibles d'endiguer une invasion; espacées de plusieurs kilomètres les unes des autres, il suffit pour les troupes et les blindés de les contourner. En revanche, elles peuvent s'avérer précieuses à deux titres : d'abord pour s'y dissimuler de manière à retarder l'avance ennemie, ensuite et surtout pour servir de points d'observation essentiels à qui y dispose des systèmes de détection perfectionnés.

7 Pour résumer notre propos, indiquons qu'à lui seul le Golan/Hermon fournit directement à Israël, entre l'affluent Banyas d'une part, les cours d'eau mineurs et nappes s'écoulant dans le Jourdain, le Yarmouk, et le lac de Tibériade d'autre part, un peu plus de 250 millions de $\mathrm{m}^{3}$ d'eau/an. Mais la dimension stratégique du plateau et du massif, intervient à plein s'agissant de la protection du Jourdain et du lac de Tibériade. Ce rôle de forteresse naturelle protectrice d'une richesse naturelle, plus encore que celui de château d'eau, confère au Golan une place prépondérante parmi les sols et territoires actuellement sous contrôle israélien.

8 Déjà en 1948, au cours de la première guerre israélo-arabe, les défenseurs juifs de Galilée, pourtant arc-boutés à seulement un contre dix autour des kibboutzim fortifiés de la vallée du Hulé et des abords du lac de Tibériade, avaient résisté victorieusement aux troupes syriennes ; l'objectif de Damas était alors de s'emparer au seul profit syrien du lac de Tibériade, de la vaste et fertile Galilée et, au mieux, du grand port méditerranéen de Haïfa. À l'automne, lorsque l'armée israélienne nouvellement constituée avait opéré une contre-offensive, les corps de troupes syriens s'étaient retranchés sur les hauteurs du Golan pour n'y plus bouger jusqu'à la signature de la convention d'armistice de Mohaynaïm, le 20 juillet 1949. Les leçons de cet échec furent méditées. Puisque l'armée syrienne disposait de cette puissante barrière naturelle en surplomb du territoire ennemi, que ne devait-elle en maximaliser l'exploitation? S'établir puissamment sur le plateau, y disposer de l'artillerie et l'aménager en base de repli truffé de casemates et de galeries offrait un avantage de taille, simultanément offensif et défensif. Depuis la crête dominant le lac de Tibériade et le Jourdain, les batteries syriennes, protégées par l'angle mort, pouvaient aisément frapper n'importe quelle installation israélienne située en contrebas sans risquer de représailles meurtrières à l'arme automatique ou au canon léger. Dès 1949, une unité de tireurs d'élite fut mise en place sur la crête et chargée, en fonction des choix politiques du moment, d'abattre des civils des kibboutzim frontaliers de Shamir, Snir, Kfar Szold et surtout Ein Guev, ce dernier étant situé à moins de 300 mètres des premiers fortins syriens.

9 L'avantage de cette tactique de harcèlement correspondait à son coût extrêmement faible tant en hommes qu'en matériel. Car pour administrer une riposte efficace, l'Étatmajor israélien ne possédait que deux uniques recours, aussi peu probants l'un que l'autre. Le premier, impensable autrement qu'à la faveur d'un conflit généralisé (tel celui de 1967), consistait à lancer une grande offensive terrestre à la conquête du 
plateau. Or dans les premières années de l'État, face à un bloc arabe uni et sans le soutien d'une grande puissance, cette solution eût été réellement irresponsable. En outre, comment justifier le déclenchement d'une guerre à outrance avec plusieurs États en guise de simple expédition punitive ? Le seul allié d'Israël dans les années 1950-60, la France, n'eut sans doute pas cautionné une entreprise d'une telle envergure.

Le second recours de Tsahal face à la tactique syrienne, à peine moins massif, était la riposte par l'aviation. Or d'une authentique aviation de combat digne de ce nom, Israël ne disposa qu'au milieu des années 1950. Par la suite, des raids de représailles après des tirs d'artillerie syriens furent en effet effectués, de plus en plus fréquemment. Mais là encore, les sorties de chasseurs-bombardiers faisaient figure de coups de massue après piqûres d'insecte, risquant à tout moment non seulement d'entraîner une condamnation des Nations Unies - où Israël était déjà fort isolé - mais encore de déclencher un conflit généralisé dont l'État juif aurait eu à assumer seul la responsabilité. La partie syrienne, à ce jeu, était donc presque toujours gagnante en poussant l'adversaire à la faute politique.

11 Enfin, autre avantage de la tactique du harcèlement à l'arme légère, les troupes de Damas pouvaient à loisir utiliser comme protections efficaces les nombreuses cavités et aspérités du plateau volcanique. Les appareils israéliens, détectés rapidement par les radars syriens disposés en altitude, ne trouvaient que peu de cibles en surface tant les retranchements étaient profonds et dissimulés. À plusieurs reprises, notamment en 1964 et 1966, Tsahal parvint à endommager sérieusement certaines installations militaires et hydrauliques. Mais sur les dix-huit années d'activités militaires syriennes sur le plateau du Golan, l'avantage demeura pour l'essentiel à Damas.

Organisé à la manière d'une forteresse, le Golan syrien tombe pourtant les 8 et 9 juin 1967 aux mains de Tsahal, suite à une série d'opérations excessivement audacieuses ; les blindés israéliens attaquent là où l'escarpement est le plus fort (nord du plateau) et donc la percée la plus improbable. Depuis, le contrôle du plateau par Tsahal permet non seulement d'observer ce qui se passe en contrebas, dans la plaine du Hauran syrien menant à Damas $(40 \mathrm{~km})$, et d'éviter ainsi tout effet de surprise en cas d'attaque terrestre ou aérienne ${ }^{9}$; ainsi le 6 octobre 1973, l'effet surprise de l'attaque blindée syrienne est considérablement atténué par le temps perdu à gravir les côtes orientales et à franchir les obstacles naturels de l'est du plateau.

Perspectives

13 Même en admettant que Damas souhaite réellement recouvrer sa souveraineté sur le plateau - ce dont nous doutons au vu de l'intérêt politique intérieur que cette « AlsaceLorraine » représente pour le pouvoir alaouite minoritaire - qu'en sera-t-il du côté israélien ? ${ }^{10}$ À l'heure où s'inscrivent ces lignes, la Knesset ne compte pas une quarantaine de voix favorables à des concessions territoriales sur le Golan, parlementaires arabes compris. Certes, les rapports de force politiques évoluent, et la situation diplomatique pourrait se débloquer en cas de rupture d'alliance entre Damas et Téhéran par exemple. Mais à deux reprises déjà, dans des contextes pourtant objectivement favorables, les Israéliens ont démontré leur attachement à la possession du plateau et, depuis les années 1990, la population juive implantée y a accru au point d'atteindre celle des Druzes: en 2007, on dénombrait ainsi environ 20000 juifs implantés sur 31 villages et une ville, Katzrin. Psychologiquement, cette nouvelle réalité démographique ajoutée à celle de la longévité - Israël contrôle le plateau depuis quarante et un ans tandis qu'il ne fut syrien que 22 années durant ! - devrait renforcer 
encore le sentiment d'un droit multiple au maintien du statu quo; ce sentiment transcende toutes les formations sionistes jusqu'à la gauche radicale du parti Meretz, et il apparaît peu vraisemblable qu'on assiste à court ou moyen terme à un retournement d'opinion sur cette question.

\section{NOTES}

1. On prononcera le $k h$ à la manière de la jota espagnole.

2. L'émission très populaire "Popolitika ", notamment, a véhiculé de longues années durant cette image dichotomique.

3. Il s'agit de Majdjal Shams, Bukhata, Massade et Eyn Qunya. Dans leur grande majorité, les 20000 Druzes du plateau ont choisi de demeurer syriens (contrairement à leurs coreligionnaires galiléens), mais tous vivent en harmonie avec les autorités et jamais depuis 1967, on n'enregistra de violences judéo-druzes.

4. Pour un approfondissement sur cet aspect mémoriel et/ou biblique, je renvoie à mon ouvrage Le Moyen-Orient entre guerre et paix. Une géopolitique du Golan, Flammarion, Champs, 2000.

5. Pour étayer ce sentiment, les juifs israéliens évoquent pêle-mêle le harcèlement frontalier des années 1948-67, les discours antisémites du président Bashar el Assad (aux côtés du pape, en 2000), et les sorts infligés aux prisonniers en 1973-74, à la communauté juive de Syrie (jusqu'en 1994), à l'espion Elie Cohen, etc.

6. Sur les aspects militaires et stratégiques, on lira avec profit Pierre Razoux : La guerre israélo-arabe d'octobre 1973. Une nouvelle donne militaire au Proche-Orient. Economica, 1999.

7. Les tendances lourdes de l'opinion israélienne sur les questions de sécurité sont analysées avec finesse par Asher Arian dans ses différents ouvrages. En l'espèce, on se reportera à Security Threatened: Surveying Israeli Opinion on Peace and War, Cambridge University Press, 1995.

8. Le parti Haderekh haShlishit, fondé en 1994, réussit à obtenir quatre sièges en 1996 et un poste ministériel (sécurité intérieure) important. Il ne survécut pas, mais un puissant lobby à la fois agricole et militaire, bien représenté à la Knesset par des députés de presque tous les partis, défend avec constance le maintien du statu quo. Reste que le livre souvenir d'Avigdor Kahalani (traduit en anglais sous le titre The Heights of Courage. A Tank Leader's War on the Golan, 1975) est encore l'un des plus lus à ce jour en Israël.

9. Depuis les années 1980, Ramat haGolan, le plateau du Golan, est truffé d'antennes couvrant la plaine syrienne du Hauran, d'où le surnom courant des « yeux d'Israël ». 10. La façon dont Damas négocia dans les années 1990 fournit une indication supplémentaire quant à cette posture. Je propose un développement de cette théorie dans mon ouvrage, Géopolitique du sionisme. Stratégies d'Israël. Armand Colin, Perspectives géopolitiques, 2006. 


\section{RÉSUMÉS}

Le plateau volcanique du Golan, dépourvu de toute ressource naturelle commercialisable et en principe situé hors des périples prophétiques, fut le théâtre de la plus grande bataille de chars depuis la seconde guerre mondiale, et le motif principal d'une alerte nucléaire entre superpuissances pendant la guerre froide. Sous souveraineté syrienne de 1945 à 1967, il fut annexé à deux reprises, fait unique, par Israël. Au sein de l'État juif, une large majorité de citoyens s'est toujours affirmée en défaveur de la restitution de cet «enfant chéri des Territoires ".

INDEX

Mots-clés : géopolitique, géographie, Proche-Orient, relations internationales, Golan, Israël

\section{AUTEUR}

\section{FRÉDÉRIC ENCEL}

Frédéric Encel, politologue, docteur en géopolitique habilité à diriger des recherches, est professeur de relations internationales à l'ESG, et maître de séminaires à l'Institut d'études politiques de Paris. Il a publié plusieurs ouvrages consacrés au Moyen-Orient et à la géopolitique. 